\title{
Apoyo a la lactancia materna en una zona básica de salud; prevalencia y factores sociosanitarios relacionados
}

\author{
MJ. Palomares Gimeno ${ }^{a}$, E. Fabregat Ferrer ${ }^{a}$, S. Folch Manuel ${ }^{b}$, \\ B. Escrig Garcíab, F. Escoín Peña ${ }^{b}$ C. Gil Segarra ${ }^{c}$

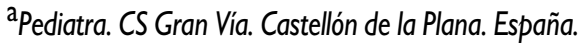 \\ benfermera. CS Gran Vía. Castellón de la Plana. España. \\ 'Matrona. CS Gran Vía. Castellón de la Plana. España.
}

\section{Resumen}

Introducción: para promocionar y apoyar la lactancia materna en nuestra población, se puso en marcha la visita prenatal en el tercer trimestre de gestación realizada por pediatras. Nuestro objetivo era valorar la duración del amamantamiento en nuestro medio y los factores sociosanitarios asociados para mejorar la salud maternoinfantil.

Material y métodos: estudio prospectivo realizado mediante encuesta presencial en el control de salud de los seis a los 15 meses, durante un periodo de dos años. Se contó con la colaboración de Enfermería Pediátrica y matronas, y con el grupo de apoyo externo "Mamare Castelló".

Resultados: prevalencia de lactancia materna completa a los seis meses del $40 \%$, y lactancia materna continuada a los 15 meses del 16\%. Los factores con relevancia estadística, desfavorables para la lactancia en el primer mes de vida, y que se mantienen al sexto mes, son ser hijo de madre fumadora, ser hijo de madre no amamantada, peso al nacimiento menor de 2500 g, iniciar la lactancia después de las cuatro horas de vida y ofrecer suero glucosado en la maternidad. Las madres inmigrantes inician lactancia materna con mayor frecuencia, pero al sexto mes no hay diferencia con las españolas.

Conclusión: las intervenciones realizadas en Atención Primaria destinadas a promover la lactancia natural consiguen resultados positivos para la salud de la población, pero se precisan mayores esfuerzos y coordinación para avanzar en la dirección propuesta por la Iniciativa para la Humanización de la Asistencia al Nacimiento para hospitales y centros de salud.

Palabras clave: Lactancia materna. Atención Primaria. Prevalencia. Factores socioeconómicos.

Ma José Palomares Gimeno, mjpalomares796x@cv.gva.es

Los autores declaran no presentar conflictos de intereses en relación con la preparación y publicación de este artículo. 

factors

Support to breastfeeding in a basic health area; prevalence and related socioeconomic

\section{Abstract}

Introduction: the prenatal visit was launched in order to promote and support breastfeeding in our population. It takes place in the third trimester of pregnancy and is carried out by pediatricians. Our objective was to assess the duration of breastfeeding in our environment and the factors related to improving mother-child health.

Material and methods: prospective study through an interview carried out during the well child visits at 6 and 15 months for a period of two years. We had the cooperation of pediatric nurses, midwives and outer-group support "Mamare Castelló".

Results: we found a $40 \%$ prevalence of full breastfeeding at 6 months and continued breastfeeding of $16 \%$ at 15 months. The factors with statistical significance against breastfeeding in the first month of life, that continue at 6th months of life are: mother who smoked, mother not breastfed herself, birth weight less than 2500 g, first breastfeeding after 4 hours of life and provide glucose solution in the Maternity. Immigrant mothers initiate breastfeeding more often but at the sixth month there are no differences with the Spanish mothers.

Conclusion: the interventions in primary care to promote breastfeeding achieve positive outcomes for the health of the population but require greater efforts and coordination to move in the direction proposed by BFHI for hospitals and health centres.

Key words: Breastfeeding. Primary care. Prevalence. Socioeconomic factors.

\section{Introducción}

Los profesionales de la salud que atendemos el embarazo y la infancia estamos cada vez más sensibilizados frente al importante problema de salud pública que supone la baja tasa de amamantamiento, tanto en cuanto al inicio como por lo que se refiere a la duración de la misma. Se ha llamado la nueva enfermedad del siglo xx-xxI y, ciertamente, la realidad española y mundial se caracteriza por el abandono progresivo de la lactancia materna (LM) a partir de la Segunda Guerra Mundial. Esta situación llevó a distintos organismos internacionales a realizar recomendaciones y planes de acción que se han ido sucediendo en las tres últimas décadas y paulatinamente han conseguido concienciar a la comunidad científica y a la sociedad de la necesidad de recuperar la cultura médica y social de la lactancia natural. La lactancia materna exclusiva (LME) hasta los seis meses de vida, y después complementada con otros alimentos adecuados y bien preparados, es la vía para conseguir un desarrollo, una salud y un crecimiento óptimos en el niño ${ }^{1-3}$.

La formación teórica y práctica de los pediatras ha mejorado gracias al trabajo de excelentes profesionales, como el Comité de Lactancia Materna de la Asociación Española de Pediatría ${ }^{4}$ y grupos de trabajo como Previnfad de la Asocia- 
ción Española de Pediatría de Atención Primaria ${ }^{5}$, que han puesto a nuestro alcance la información más relevante y actualizada, accesible desde cualquier consulta de Atención Primaria (AP), gracias a Internet, para incorporarla a nuestra práctica clínica 6 .

Como se ha demostrado durante los últimos años, las intervenciones de promoción y apoyo a la LM realizadas desde AP han sido efectivas para aumentar la duración tanto a corto como a largo plazo. Los resultados mejoran si se combinan actuaciones pre- y postparto e intervienen distintos profesionales ${ }^{7-10}$. Así pues, decidimos instaurar la visita prenatal en el tercer trimestre de la gestación, realizada por el pediatra, acción recomendada por Previnfad desde el año $2003^{11}$, aunque no estuviera incluida en nuestra cartera de servicios. Se entregó material escrito al finalizar la entrevista (disponible en www.aeped/lactancia materna) y se animó a solicitar cita pre$\mathrm{COz}$, si surgían dificultades tras el alta hospitalaria, con la enfermera de Pediatría, que habitualmente realiza la primera visita de acogida a los 5-7 días. El equipo materno-infantil está formado por dos pediatras, dos enfermeras y una matrona (compartida con otro centro) que realiza la preparación al parto, posterior visita de seguimiento en el puer- perio y sesiones de masaje infantil que servían como un verdadero taller de lactancia. Además, contamos con la colaboración de un grupo de apoyo local ${ }^{12}$, con sede en nuestro propio centro de salud. Nuestro objetivo era valorar la duración del amamantamiento en nuestro medio y conocer los factores sociosanitarios asociados para mejorar la salud materno-infantil. Al no disponer de un sistema oficial de monitorización que nos permitiera registrar y recuperar los datos de prevalencia de la LM, acordamos utilizar la clasificación internacional de enfermedades CIE-9, que es la que incorpora nuestro programa Abucasis de la Agència Valenciana de Salut (AVS) y, aprovechando la posibilidad que ofrece el sistema de personalizar diagnósticos, escogimos tres de ellos y se los atribuimos al lactante según fuera su alimentación: $L M$ completa $(L M C)$, mixta o parcial (LMP) y artificial (LA), para facilitarnos el seguimiento y la recogida posterior de datos.

\section{Material y métodos}

Estudio descriptivo, longitudinal y prospectivo sobre la prevalencia de la LM, su inicio y su duración, y la valoración de factores que pueden influir sobre ella, con seguimiento hasta los 15 meses de vida. Se trata de un estudio observa- 
cional y, aunque se ha realizado un análisis bivariante, nos hemos apoyado en otros que realizan análisis multivariante ${ }^{13-15}$.

La población objeto de estudio incluyó a todos los nacidos entre el 1 de abril de 2007 y el 31 de marzo de 2009 asignados al centro de salud Gran Vía de Castellón. Fueron 361 niños, distribuidos en dos cupos de Pediatría. Nuestro centro, situado en una zona de expansión de la ciudad, capital de provincia de unos 180000 habitantes, atiende a una población de 11000 usuarios, población activa joven en su mayoría, como refleja el predominio de la franja de edad entre 30 y 35 años en la pirámide poblacional.
La recogida de datos para el estudio la realizó mediante encuesta presencial (tabla 1) la enfermera de Pediatría durante el control de salud de los seis meses. A las madres que seguían amamantando se les preguntó en el control del año hasta qué mes habían continuado con la LM y se repitió la misma pregunta a las que continuaban en el control de los 15 meses.

Las pediatras revisaron los datos de la historia clínica informatizada utilizando el sistema de monitorización ideado ad hoc para confrontar los datos. Se escogió siempre el dato de menor edad de abandono de la LM.

A las madres que habían optado por dar LA en la maternidad no se las inte-

\begin{tabular}{|c|c|}
\hline \multirow[t]{7}{*}{ Datos de la madre } & 1. País de origen \\
\hline & 2. Edad de la madre al parto \\
\hline & 3. Situación laboral: en casa/trabaja fuera \\
\hline & 4. Es fumadora habitual: sí/no \\
\hline & 5. Nivel estudios: primarios/secundarios/universidad \\
\hline & 6. ¿Usted recibió lactancia natural al nacimiento? Sí/no \\
\hline & $\begin{array}{l}\text { 7. ¿Recibió información prenatal de la lactancia por matrona, } \\
\text { pediatra o grupos de apoyo? Sí/no }\end{array}$ \\
\hline \multirow[t]{6}{*}{ Datos perinatales } & 8. Sexo \\
\hline & 9. Peso al nacimiento $(\mathrm{g})$ \\
\hline & 10. Tipo de parto: vaginal/cesárea \\
\hline & 11. Días de estancia del bebé en el hospital \\
\hline & 12. ¿Se le ofreció agua o suero al bebé en la maternidad? Sí/no \\
\hline & 13. ¿Cuántos minutos después del parto inició el amamantamiento? \\
\hline \multirow[t]{3}{*}{ Datos de alimentación } & 14. Meses de lactancia materna exclusiva \\
\hline & $\begin{array}{l}\text { 15. Edad en meses de introducción del primer biberón de leche } \\
\text { adaptada }\end{array}$ \\
\hline & 16. Edad en meses de abandono lactancia materna \\
\hline
\end{tabular}


rrogó sobre las cuestiones que no eran pertinentes (últimas cinco preguntas) para evitar que se sintieran culpabilizadas. Las variables 15 y 16 son reiterativas como control de veracidad de la respuesta de la madre.

Adoptamos los indicadores de LM de la Organización Mundial de la Salud $(\mathrm{OMS})^{16}$ : LMC cuando el bebé era alimentado exclusivamente con leche materna (con o sin pequeñas cantidades de otros líquidos no lácteos, agua o infusiones), incluyendo así tanto la LME como la predominante. Clasificamos dentro del grupo LA a los lactantes que recibían alimentación con fórmulas lácteas, pero que no recibían leche materna, independientemente de si recibían cualquier otro alimento. Aquellos niños que recibían simultáneamente leche materna y leche artificial con o sin otros alimentos fueron clasificados en el grupo de LMP.

Los datos se recogieron en programa Excel $^{\circledR}$ y se utilizaron estadísticos descriptivos: media, desviación estándar, intervalos de frecuencias; y análisis estadístico: odds ratio (OR) con su intervalo de confianza (IC 95\%), test chi-cuadrado para variables cualitativas, y $t$ de Student para variables cuantitativas.

\section{Resultados}

\section{Descripción de la muestra}

Se completaron 335 encuestas, correspondientes al 92,8\% de la población asignada. Las pérdidas fueron escasas, solo 26 niños: unos por no utilizar el sistema sanitario público pese a tener su domicilio en nuestra zona básica de salud (posibles usuarios de aseguradoras privadas), y otros por cambio de domicilio antes de los 15 meses (algunos de ellos inmigrantes que han regresado a su país de origen).

La edad de las madres osciló entre los 17 y los 43 años, con una media de 30,9 años. El $26,9 \%$ de las madres era de origen extranjero; el 25\% había cursado estudios primarios; el $47,5 \%$, secundarios, y el 27,5\% tenía formación universitaria. El $60,6 \%$ tenía un trabajo remunerado fuera de casa. El $26,6 \%$ declaró fumar habitualmente. El $91,6 \%$ de las entrevistadas refería haber recibido información sobre los beneficios de la LM antes del parto y el 71,3\% había sido amamantado por sus madres (actuales abuelas).

Los lactantes varones fueron un $49,5 \%$ y las niñas un $50,5 \%$. Los nacimientos por cesárea supusieron el $26,3 \%$ del total. La media de peso al nacer fue de $3182 \mathrm{~g}$. Los de peso bajo representaron 


\begin{tabular}{l|c|c|c}
\hline \multicolumn{4}{l}{ Tabla 2. Datos de prevalencia. Lactancia por edad en la población estudiada } \\
\hline Edad (meses) & LMC (\%) & LMP (\%) & LA (\%) \\
\hline 0 & 81,3 & - & 18,7 \\
\hline 1 & 65,1 & 10,4 & 24,5 \\
\hline 2 & 59,4 & 9,3 & 31,0 \\
\hline 3 & 55,2 & 11,7 & 36,4 \\
\hline 4 & 51,9 & 6,7 & 41,2 \\
5 & 45,7 & 9,2 & 45,1 \\
\hline 6 & 40,0 & 11,9 & 48,1 \\
\hline 7 & 33,1 & 11,3 & 55,5 \\
\hline 8 & 26,6 & 12,3 & 61,2 \\
\hline 9 & 22,7 & 10,4 & 66,9 \\
10 & 20,0 & 9,8 & 70,1 \\
\hline 11 & 16,1 & 9,5 & 74,3 \\
\hline 12 & 14,6 & 9,2 & 76,1 \\
\hline 13 & 11,2 & 7,4 & 81,2 \\
\hline 14 & 10,7 & 6,9 & 82,4 \\
\hline 15 & 10,4 & 6,3 & 83,3 \\
\hline
\end{tabular}

LA: Iactancia artificial; LMC: lactancia materna completa; LMP: lactancia materna parcial o mixta.

Figura 1. Distribución de tipos de lactancia desde el nacimiento hasta los 15 meses de edad en la población estudiada.

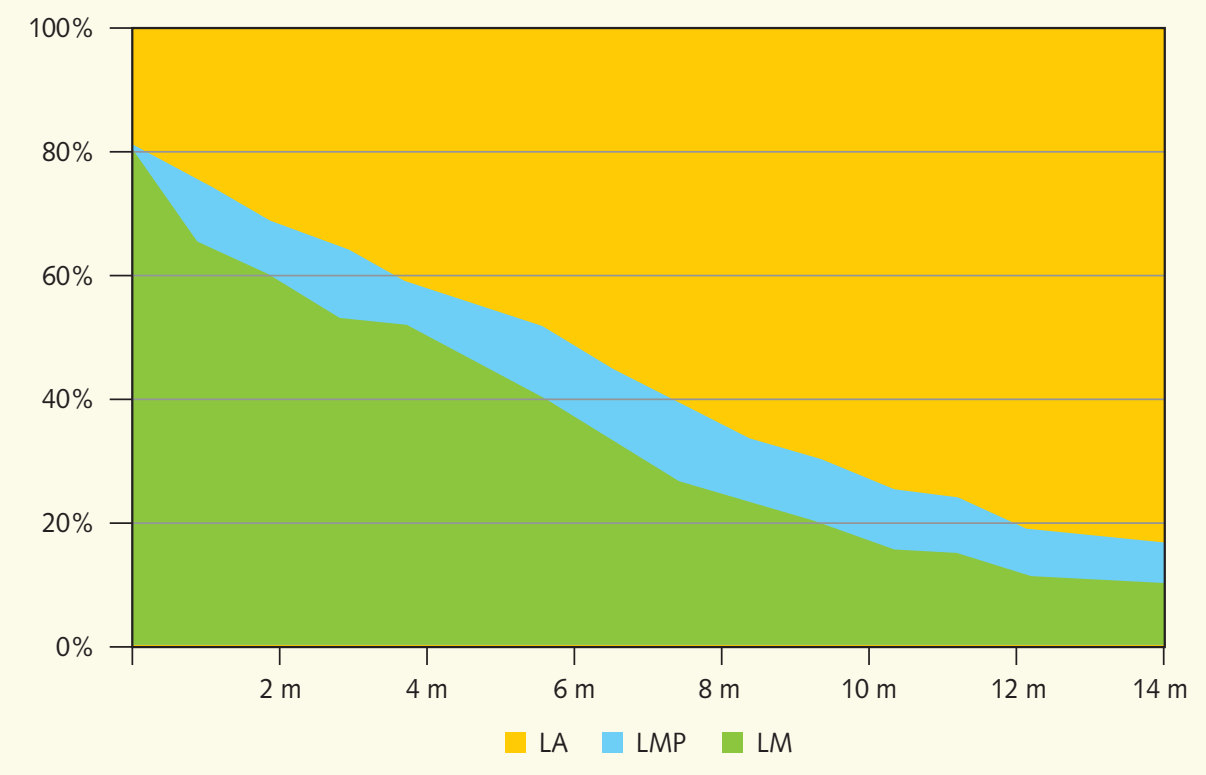

LA: lactancia artificial; LMC: lactancia materna completa; LMP: lactancia materna parcial o mixta. 
un $9,5 \%$ de la muestra y un $5 \%$ fueron macrosómicos, de más de $4 \mathrm{~kg}$.

La estancia media en el hospital fue de cuatro días y la moda tres días.

Un 18,8\% había decidido alimentar a su hijo con sucedáneos y el 81,2\% inició LM. De estas, el $69,8 \%$ se puso al bebé al pecho antes de los 30 minutos de vida, el $16,9 \%$ entre los 30 minutos y las cuatro horas, y el $13,2 \%$ transcurridas más de cuatro horas desde el nacimiento. Un $34,8 \%$ de los bebés amamantados recibió agua o suero glucosado en la maternidad.

\section{Lactancia materna}

Un $81,2 \%$ de las madres inició lactancia natural tras el parto. En cuanto a la prevalencia del amamantamiento (tabla 2), al mes vida un 65,1\% de los lactantes tomaba LMC, este porcentaje se redujo al 55,2\% a los tres meses y al $40 \%$ a los seis meses. $A$ los 15 meses de vida, un $16,7 \%$ de los niños se mantenía con LM (figura 1).

Al comparar el grupo de lactantes que al mes de vida recibía $L M C$ respecto a los otros dos tipos de alimentación, las variables con diferencias que resultan estadísticamente significativas (tabla 3 ) respecto a la madre son el trabajo remunerado, el hábito tabáquico y la experiencia de haber sido amamantada; res- pecto a los datos perinatales, hay diferencias estadísticamente significativas en el peso del recién nacido (RN), el tipo de parto, ofrecer o no suero glucosado en la maternidad y el inicio precoz o tardío del amamantamiento. En general, se observa una mayor contribución en las variables situadas en el eje negativo: madre fumadora, madre que no fue amamantada, haber recibido suero glucosado o agua en la maternidad y realizar la primera toma al pecho después de las cuatro horas de vida.

Realizando el mismo estudio comparativo a los lactantes que a los seis meses de vida recibían $L M C$, respecto a los que reciben $L M P$ o $L A$, vemos que siguen existiendo diferencias significativas, al igual que en el primer mes de vi$\mathrm{da}$, en las siguientes variables (tabla 4): hábito tabáquico de la madre, experiencia de haber sido amamantada, peso del $\mathrm{RN}$, ofrecer suero en la maternidad y tiempo de inicio del amamantamiento. Un dato que varía es que, a los seis meses, entre las mujeres que amantan a sus hijos predominan las que tienen estudios universitarios, con diferencia estadísticamente significativa. Sin embargo, no hay diferencias entre las madres que trabajan fuera de casa o no, ni entre las que han tenido parto vaginal o cesárea. 
Tabla 3. Probabilidad de ser alimentado con lactancia materna frente a lactancia artificial o mixta el primer mes de vida

\begin{tabular}{|c|c|c|c|c|}
\hline \multirow{2}{*}{ Datos de la madre } & \multirow[b]{2}{*}{ Trabajo } & \multirow[t]{2}{*}{ OR } & \multirow[t]{2}{*}{ IC $95 \%$} & \multirow{2}{*}{$\frac{p \text { (chi-cuadrado) }}{0,0350}$} \\
\hline & & & & \\
\hline & - En casa & 1,64 & $1,03-2,63$ & \\
\hline & - Fuera de casa & 0,60 & $0,38-0,96$ & \\
\hline & Hábito tabáquico & & & $<0,0001$ \\
\hline & - Sí fumadora & 0,32 & $0,25-0,19$ & \\
\hline & - No fumadora & 3,09 & $1,87-5,11$ & \\
\hline & Estudios & & & 0,0684 \\
\hline & - Universitarios & 1,62 & $0,96-2,73$ & \\
\hline & - Otros estudios & 0,61 & $0,36-1,03$ & \\
\hline & Alimentación recibida por la madre & & & $<0,0001$ \\
\hline & - Lactancia natural & 2,68 & $1,64-4,36$ & \\
\hline & - Leche artificial & 0,37 & $0,22-0,60$ & \\
\hline \multirow[t]{18}{*}{ Datos perinatales } & Niños/niñas & 1,25 & $0,80-1,96$ & 0,311 \\
\hline & Peso al nacer & & & 0,0128 \\
\hline & $\bullet<2500 \mathrm{~g}$ & 0,41 & $0,20-0,84$ & \\
\hline & $\cdot>2500 \mathrm{~g}$ & 2,43 & $1,18-4,99$ & \\
\hline & Tipo de parto & & & 0,0209 \\
\hline & - Vaginal & 1,79 & $1,08-2,93$ & \\
\hline & - Cesárea & 0,55 & $0,34-0,91$ & \\
\hline & Suero glucosado o agua & & & $<0,0001$ \\
\hline & • Sí & 0,18 & $0,09-0,33$ & \\
\hline & - No & 5,46 & $2,94-10,15$ & \\
\hline & Días de estancia en el hospital & & & 0,0730 \\
\hline & $\bullet>5$ días & 0,53 & $0,26-1,06$ & \\
\hline & $\bullet \leq 5$ días & 1,87 & $0,93-3,75$ & \\
\hline & Inicio del amamantamiento & & & 0,0031 \\
\hline & - Antes de 30 min de vida & 2,42 & $1,33-4,40$ & \\
\hline & - Después de 30 min & 0,41 & $0,22-0,74$ & \\
\hline & - Antes de 240 min & 4,94 & $2,43-10,05$ & \\
\hline & - Después de 240 min & 0,20 & $0,09-0,41$ & \\
\hline
\end{tabular}

IC: intervalo de confianza; min: minutos; OR: odds ratio.

\section{Madres inmigrantes}

De las madres entrevistadas, un $26,9 \%$ es inmigrante $(n=90)$, en su mayoría proceden de Rumanía $(n=46)$, seguido de América latina $(n=18)$, países del norte de África $(n=14)$, África subsahariana $(n=9)$, y otros.
La tabla 5 recoge las diferencias estadísticas encontradas entre mujeres españolas e inmigrantes en diversos factores analizados. Se evidencia que las madres inmigrantes son significativamente más jóvenes que las españolas, trabajan fuera de casa en menor medida y tienen un 
Tabla 4. Probabilidad de ser alimentado con lactancia materna frente a lactancia artificial o mixta al sexto mes de vida

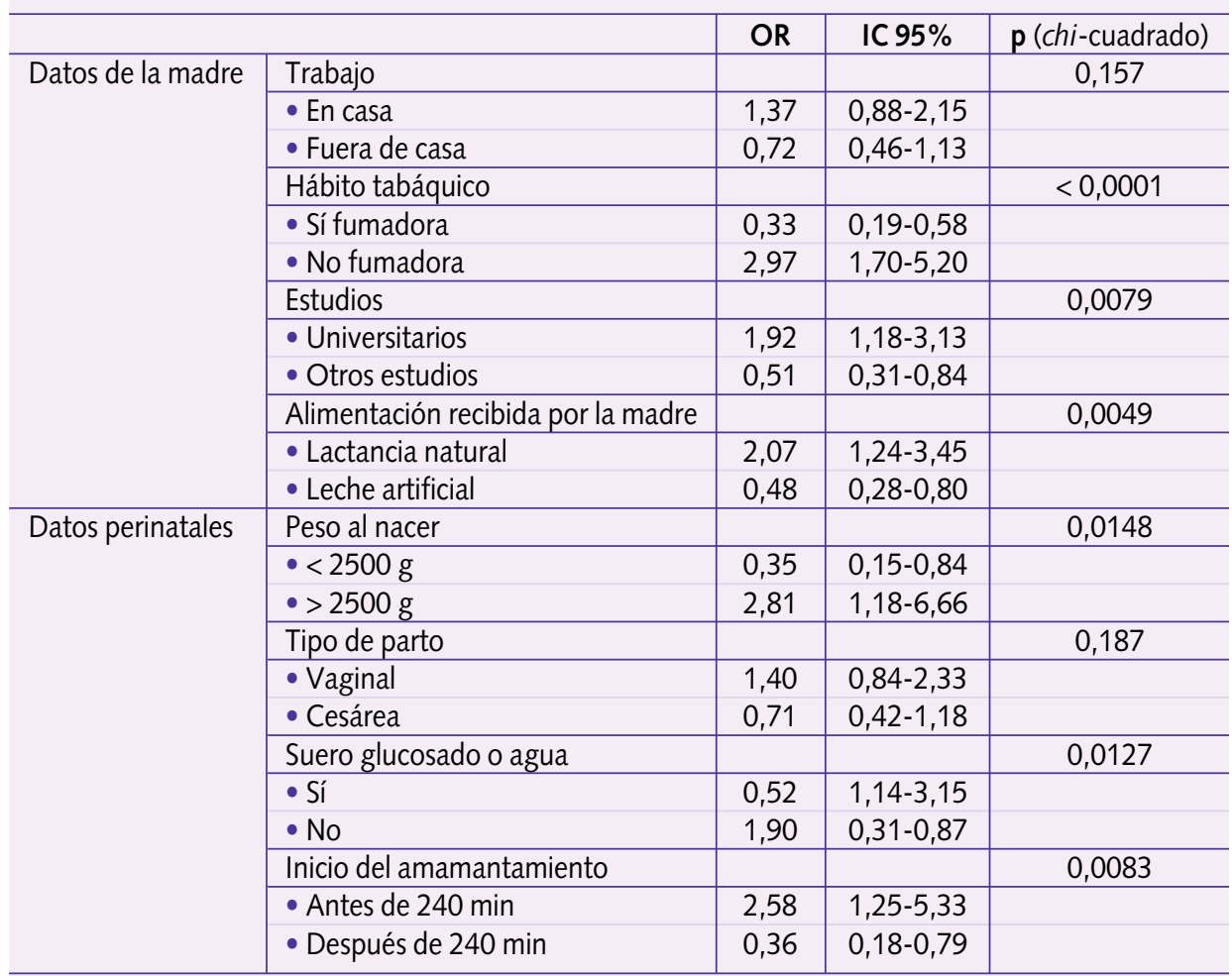

IC: intervalo de confianza; min: minutos; OR: odds ratio.

nivel de estudios inferior. Respecto a datos sanitarios, tienen menos partos por cesárea y alimentan con LMC a sus hijos en mayor proporción que las españolas un mes después del parto, pero esta diferencia ya no se observa a los seis meses de vida.

\section{Discusión y conclusiones}

La encuesta se realizó en el control de salud de los seis meses para conocer la prevalencia de LMC a esta edad como objetivo principal. Reconocemos el sesgo que puede haber al extraer datos de prevalencia intermedia al mes y a los tres meses de esta encuesta, confiando en lo que la madre recuerda o afirma y, para paliarlo en cierta medida, los pediatras contrastamos la información ofrecida por la madre con los diagnósticos adaptados para monitorizar la lactancia, tal y como hemos referido en la introducción. 


\begin{tabular}{|c|c|c|c|c|c|}
\hline \multicolumn{6}{|l|}{ Variables cuantitativas } \\
\hline Autóctonas/inmigrantes & Media & Media & \multicolumn{2}{|c|}{ T de Student IC 95\% } & $p$ \\
\hline Edad de la madre & 31,99 & 28,12 & \multicolumn{2}{|c|}{$2,80-4,93$} & $<0,0001$ \\
\hline Peso del recién nacido $(\mathrm{g})$ & 3160,00 & 3242,28 & \multicolumn{2}{|c|}{$-224,04-59,47$} & 0,2544 \\
\hline \multicolumn{6}{|l|}{ Variables cualitativas } \\
\hline \multicolumn{2}{|l|}{ Autóctonas/inmigrantes } & OR (IC 95\%) & OR (IC 95\%) & \multicolumn{2}{|c|}{ p (chi-cuadrado) } \\
\hline Madres fumadoras & \multicolumn{2}{|c|}{$\begin{array}{c}1,63 \\
(0,66-2,03)\end{array}$} & $\begin{array}{c}0,85 \\
(0,49-1,49)\end{array}$ & \multicolumn{2}{|c|}{0,5939} \\
\hline \multirow[t]{2}{*}{ Madres con trabajo fuera de casa } & \multicolumn{2}{|c|}{4,48} & 0,22 & \multirow{2}{*}{\multicolumn{2}{|c|}{$<0,0001$}} \\
\hline & \multicolumn{2}{|c|}{$(2,68-7,49)$} & $(0,13-0,37)$ & & \\
\hline \multirow[t]{2}{*}{ Madres con estudios universitarios } & & 2,76 & 0,36 & \multirow{2}{*}{\multicolumn{2}{|c|}{0,0015}} \\
\hline & \multicolumn{2}{|c|}{$(1,44-5,27)$} & $(0,18-0,68)$ & & \\
\hline \multirow[t]{2}{*}{ Madres que recibieron lactancia natural } & &, 12 & 8,12 & \multirow{2}{*}{\multicolumn{2}{|c|}{$<0,0001$}} \\
\hline & \multicolumn{2}{|c|}{$(0,05-0,29)$} & $(3,41-19,36)$ & & \\
\hline \multirow[t]{2}{*}{ Cesárea } & & 3,29 & 0,30 & \multirow{2}{*}{\multicolumn{2}{|c|}{0,0004}} \\
\hline & \multicolumn{2}{|c|}{$(1,65-6,53)$} & $(0,15-0,60)$ & & \\
\hline Lactancia materna un mes posparto & & $\begin{array}{l}0,46 \\
7-0,80)\end{array}$ & $\begin{array}{c}2,13 \\
(1,23-3,67)\end{array}$ & \multicolumn{2}{|c|}{0,0058} \\
\hline Lactancia materna seis meses posparto & & $\begin{array}{l}0,77 \\
7-1,29)\end{array}$ & $\begin{array}{c}1,28 \\
(0,78-2,09)\end{array}$ & \multicolumn{2}{|c|}{0,314} \\
\hline
\end{tabular}

No obstante, el dato de prevalencia al sexto mes se ha recogido cumpliendo los requisitos que la OMS aconseja para investigar en LM, basándose en los alimentos ingeridos las 24 horas previas.

Siguiendo la secuencia temporal desde el nacimiento, tenemos casi un $20 \%$ de madres que ya ha decidido no lactar. Para rebajar esta cifra se necesita, además del esfuerzo de los profesionales implicados, el apoyo decidido de la Administración a través de políticas informativas, laborales y sociales para generar un ambiente más proclive a la lactancia natural en la sociedad.
Del $81 \%$ que quiere amamantar, existe una parte que ya sale del hospital con lactancia mixta, pese a darse el alta cada vez más precoz. Pensamos que debería especificarse cuál ha sido el motivo para indicar el complemento, enseñar formas alternativas para no ofrecerlo con biberón y concertar una cita en AP en 24-48 horas, para retirarlo si ya no fuera necesario. Respecto a otras prácticas en la maternidad, se ha ido implantando la puesta al pecho precoz, como demuestra el dato positivo de un $70 \%$ de niños que la realizan en los primeros 30 minutos de vida, pero lamentablemente per- 
sisten rutinas contrarias a los estándares recomendados y aún se ofrece suero glucosado o agua a un tercio de los bebés que toman lactancia natural ${ }^{17,18}$.

El porcentaje de cesáreas en nuestra serie es del $26,2 \%$. Se ha demostrado que la cesárea dificulta el inicio de la lactancia ${ }^{19}$, pero parece difícil reducir este porcentaje habida cuenta del número cada vez mayor de embarazos de riesgo. Este grupo de madres tiene un alto riesgo de abandono precoz, probado por el dato de un 53\% de LMC al mes en nacidos por cesárea frente al $67 \%$ de LMC entre los nacidos por parto natural. Esta diferencia ya no se encuentra a los seis meses. En la bibliografía hay alguna iniciativa aislada que intenta conseguir una "cesárea natural" para interferir lo mínimo con la lactancia ${ }^{20}$, pero parece que, por ahora, estos intentos resultan meramente anecdóticos.

Durante las cuatro primeras semanas tenemos un $15 \%$ de abandonos. Es un

Figura 2. Datos comparativos de prevalencia de lactancia materna completa (\%) de nuestro estudio con datos de la Comunidad Valenciana y de España.

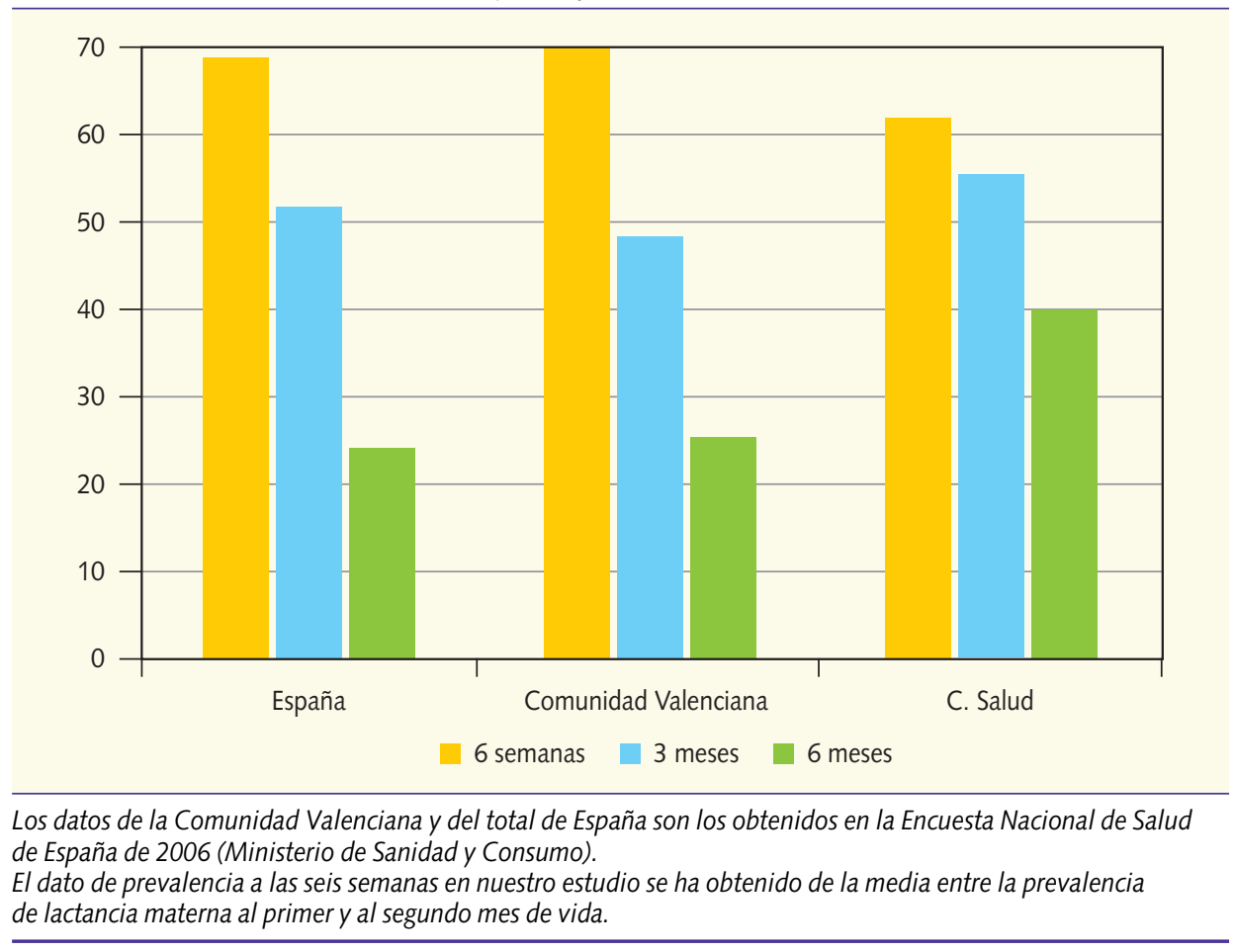


periodo crítico y aquí es donde los profesionales de AP tenemos que realizar nuestra tarea sin ahorrar esfuerzos para apoyar a las madres, promover la lactancia y considerar cualquier consulta o duda sobre la lactancia como una verdadera urgencia no demorable. Nuestros datos al mes, $65 \%$ de LMC y otro $10 \%$ de LMP, se acercan a la media nacional publicada en la Encuesta Nacional de Salud en $2006^{21}$ (figura 2) y un poco por debajo del $69,6 \%$ de la media en la Comunidad Valenciana, pero aún bastante lejos de las cifras de Aragón, Navarra, País Vasco y Rioja, que rondan el $80 \%$. No teníamos datos de prevalencia antes de iniciar la visita prenatal, así que no podemos constatar si ha sido efectiva en alguna medida, aunque creemos que todos los esfuerzos suman.

A los tres meses, los bebés con LMC son el $50 \%$, en la misma línea de la media nacional y de nuestra comunidad, y a los seis meses encontramos un $40 \%$ de niños con LMC y un $10 \%$ adicional de LMP. Este dato, bastante superior al $25 \%$ de la media nacional y comparable a las cifras de las comunidades de prevalencias más altas (noreste de España), nos sorprende gratamente y pone de manifiesto el esfuerzo realizado por el equipo por aumentar la duración del amamantamiento ${ }^{22}$. Una circunstancia que pensamos que ha influido muy favorablemente es el hecho de contar con el Grupo de Apoyo "Mamare Castelló" creado en 2004 por personal no sanitario que realiza una encomiable labor en nuestra ciudad; se reúne semanalmente en las instalaciones de nuestro centro y realiza talleres quincenales, además de otras actividades divulgativas como una exposición fotográfica itinerante que recorre diferentes centro sanitarios y que albergó el nuestro durante el periodo de estudio. El grupo está abierto a todas las madres de la ciudad que deseen acudir pero, indudablemente, la accesibilidad para nuestra población es mayor y las buenas relaciones que mantenemos con el grupo hacen que se obtengan resultados más satisfactorios.

Una importante dificultad que encuentran las madres que desean lactar en exclusiva hasta el sexto mes es la duración de la baja maternal, que en España es de 16 semanas, con lo que escasamente alcanza al cuarto mes de vida del lactante. Esos meses han de afrontarse con mucho voluntarismo porque no es fácil ni habitual disponer de leche almacenada para cubrir todas las tomas que el bebé demanda mientras su madre está ausente. Aquí nos hemos adaptado a las necesidades y deseos de las madres $y$ bastantes veces hemos recurrido a la in- 
troducción de sólidos no lácteos a partir del quinto mes.

A los 12 meses encontramos un $14,6 \%$ de niños cuya única fuente de lácteos es la LM y a los 15 meses tenemos un $10,4 \%$ de LM continuada.

Respecto a los factores estudiados relacionados con la lactancia, destacar que durante estos dos años la afluencia de población inmigrante a nuestra ciudad ha sido muy alta, como demuestra el hecho de tener más de un $27 \%$ de madres extranjeras. La incorporación de Rumanía y Bulgaria a la Unión Europea en 2007, con la apertura de fronteras, supuso una afluencia masiva a nuestra ciudad que ha sido tradicional destino de inmigración de Europa del Este. Por eso recogimos el país de origen junto con otros datos como edad de la madre, nivel de estudios, situación laboral, tabaquismo y experiencia de haber sido amamantada, para trazar un perfil sociosanitario de las madres. Los resultados que hemos obtenido indican que hay diferencias estadísticamente significativas en la edad, el trabajo remunerado y el antecedente de haber sido amamantadas por sus propias madres. Las madres inmigrantes son de media cuatro años más jóvenes, tienen menos trabajo remunerado y recibieron LM por sus madres en una proporción mucho más alta que las españolas. Estos dos últimos datos se comportan como importantes factores de riesgo para no iniciar la lactancia o abandonarla al mes del nacimiento. Las madres que trabajan y no recibieron pecho en su niñez dan menos de mamar y eso se comprueba tanto en las españolas como en las extranjeras. Entre estas, las cesáreas fueron bastante menos frecuentes, dato que no sabemos cómo interpretar, pues podría deberse a que son madres más jóvenes y quizás con menos problemas perinatales. En la literatura médica existen datos contradictorios respecto a la influencia del estatus socioeconómico sobre el fenómeno de la lactancia, unos encuentran que a medida que aumenta el grado de "culturización" la lactancia disminuye ${ }^{23}$ (EE. UU.) y otros demuestran que cuanto mayor es el estatus socioeconómico más se favorece la lactancia ${ }^{24}$. Nos ha parecido muy interesante la publicación sobre lactancia en Irán, donde hay una serie de provincias con diferente desarrollo económico y una implantación en el $100 \%$ de hospitales de la Iniciativa para la Humanización de la Asistencia al Nacimiento (IHAN) y parece que, finalmente, donde los datos son mejores es en la comarca donde hay una importante tradición de amamantar ${ }^{25}$. Esta circunstancia podría explicar por qué la población inmigrante 
da pecho con mayor frecuencia al mes de vida pero a los seis meses se comporta igual que la población autóctona.

El tabaquismo materno es un claro factor desfavorable para iniciar y mantener la LM; así pues, el hijo de madre fumadora tiene más morbilidad por exposición al humo del tabaco y por carecer de la protección de la leche materna ${ }^{26,27}$. Este es otro grupo de madres de riesgo que debemos identificar y proponernos como objetivo conseguir que, aunque no puedan dejar de fumar, amamanten a sus bebés.

Respecto al trabajo fuera de casa, ya hemos comentado que las madres comienzan menos la lactancia pero, una vez establecida, la mantienen a los seis meses en igual medida que las que no trabajan. Las madres con estudios universitarios mantienen más tiempo la lactancia que las demás. Parece que madres de características socioculturales distintas se comportan de forma parecida frente al fenómeno de la lactancia.

\section{Conclusiones}

En nuestra zona básica de salud encontramos una prevalencia de LM similar a la media nacional al mes y a los tres meses, pero bastante superior a los seis meses de vida, lo cual atribuimos al trabajo conjunto de los profesionales de
AP, maternidad y grupos de apoyo externos.

Los factores de riesgo para el abandono de la LM por parte de la madre han sido el tabaquismo y no haber sido amamantada en su infancia; este último hecho es reflejo de la pérdida de la cultura de la lactancia que afectó a la generación de las actuales abuelas y que puede compensarse incorporándolas a las actividades de promoción y apoyo durante el embarazo, el parto y las primeras semanas tras el nacimiento.

El tabaquismo es una adicción difícil de combatir, máxime si no se ha conseguido ya durante la gestación, pero estos niños necesitan aún más que otros los beneficios de la LM y nuestra obligación es poner todos los medios a nuestro alcance para que lo consigan.

Los factores de riesgo perinatales son el bajo peso al nacimiento, la puesta tardía al pecho y recibir complementos como agua o suero glucosado. Todos ellos pueden mejorarse desde las maternidades mediante una rigurosa aplicación de las prácticas y los estándares recomendados para el cuidado del RN.

La extensión fuera de las maternidades de la IHAN ha implicado a los centros de salud ${ }^{28}$ en este proyecto, lo que nos afecta a todos y nos permite trabajar de una forma más sistemática, ha- 
ciendo que nos sintamos parte de un proyecto global. Desde septiembre de 2009 se ha lanzado en España esta iniciativa, que incluye siete puntos, de los que hemos intentado aplicar el tercero (información a las madres previa al par- to), el sexto (atmósfera receptiva a la madre que amamanta) y el séptimo (colaboración con los grupos de apoyo), y esperamos seguir avanzando para mejorar la salud de los niños y de toda la población.

\section{Bibliografía}

1. Hernández Aguilar MT, Aguayo Maldonado J. La lactancia materna. Cómo promover y apoyar la lactancia materna en la práctica pediátrica. Recomendaciones del Comité de Lactancia Materna de la AEP. An Pediatr (Barc). 2005;63:340-56.

2. Gartner LM, Morton J, Lawrence RA, Naylor AJ, O'Hare D, Schanler RJ et al. Breastfeeding and the use of human milk. Pediatrics. 2005;115:496506.

3. Aizpurua Galdeano P. Efectos de la lactancia materna sobre la salud de las madres y de los lactantes en países desarrollados. Evid Pediatr. 2008; 4:45. Traducción autorizada de: Breastfeeding and maternal and infant health outcomes in developed countries University of York. Centre of Reviews and Dissemination (CRD). Database of Abstracts of Review of Effects (DARE) [consultado el 28/04/ 2008]. Disponible en www.crd.york.ac.uk/CRDWeb/Show Record.asp?View=Full\&ID=32007000496

4. Comité de lactancia materna de la Asociación Española de Pediatría. Manual de Lactancia Materna. De la teoría a la práctica. Madrid: Editorial Médica Panamericana; 2008.

5. Pallás Alonso CR. Promoción de la lactancia materna. Recomendaciones PrevInfad/PAPPS. Actualizado diciembre de 2006 [consultado el 20/07/2010]. Disponible en www.aepap.org/ previnfad/rec_lactancia.htm
6. Hernández Aguilar MT, Cerveró L, García Ballester M, Fernández Pérez M, Gutiérrez G, Lloret J y cols. Manejo de la lactancia materna desde el embarazo hasta el segundo año. Guía de práctica clínica basada en la evidencia [consultado el 20/07/2010]. Disponible en www.aeped.es/ sites/default/files/Im_gpc_peset_2004.pdf

7. Aparicio Rodrigo $M$, Balaguer Santamaría $A$. Con breves sesiones de educación sanitaria pueden aumentarse las tasas de lactancia materna exclusiva. Evid Pediatr. 2007;3:93.

8. U.S. Preventive Services Task Force. Primary care interventions to promote breastfeeding: U.S. Preventive Services Task Force Recommendation Statement. Ann Intern Med. 2008;149:560-4.

9. Chung M, Raman G, Trikalinos T, Lau J, Ip S. Interventions in primary care to promote breastfeeding: an evidence review for the U.S. preventive services task force. Ann Intern Med. 2008;149:565-82.

10. Labarere J, Gelbert-Baudino N, Ayral AS, Duc C, Berchotteau M, Bouchon N et al. Efficacy of breastfeeding support provided by trained clinicians during an early, routine, preventive visit: a prospective, randomized, open trial of 226 mother-infant pairs. Pediatrics. 2005;115:e139-46.

11. Pallás Alonso CR. Prevención prenatal. Visita prenatal. En Recomendaciones Previnfad/PAPPS. Actualizado octubre de 2005 [consultado el 20/07/2010]. Disponible en www.aepap.org/ previnfad/prenatal.htm. 
12. Associació de suport a la lactància materna. Mamare Castelló. Grupo de apoyo a la lactancia [consultado el 20/07/2010]. Disponible en www. mamare.es

13. Hernández Aguilar MT, Muñoz Guillén $A$, Lasarte Velillas JJ, García Vera C, Díaz Marijuan MC, Martín Calama J. La lactancia materna en la Comunidad Valenciana. Análisis multivariante de una encuesta a 6400 lactantes. Rev Pediatr Aten Primaria. 2004;6:19-37.

14. Barriuso Lapresa LM. Estudio multivariante sobre la prevalencia de la lactancia materna en el centro-norte de España. Rev Pediatr Aten Primaria. 2007;9:589-612.

15. Sánchez Bayle M, Cano Fernández C, García García MC, Yep Chullen G, Pérez Suárez E. Inmigración, lactancia materna y hábito tabáquico. An Pediatr (Barc). 2008;68:462-5.

16. World Health Organization. Indicadores para evaluar las prácticas de alimentación del lactante y del niño pequeño: conclusiones de la reunión de consenso llevada a cabo del 6 al 8 de noviembre de 2007 en Washington, DC, EE. UU. Ginebra: Ediciones de la OMS; 2009.

17. Academy of Breastfeeding Medicine Protocol Committee. ABM clinical protocol \#3: hospital guidelines for the use of supplementary feedings in the healthy term breastfed neonate, revised 2009. Breastfeed Med. 2009;4:175-82.

18. Sánchez Luna $M$, Pallás Alonso $C R$, Botet Mussons F, Echániz Urcelay I, Castro Conde JR, Narbona E y Comisión de Estándares de la Sociedad Española de Neonatología. Recomendaciones para el cuidado y atención del recién nacido sano en el parto y en las primeras horas después del nacimiento. An Pediatr (Barc). 2009;71:349-61.

19. Rowe-Murray HJ, Fisher JR. Baby friendly hospital practices: cesarean section is a persistent barrier to early initiation of breastfeeding. Birth. 2002;29:124-31.
20. Smith J, Plaat F, Fisk N. The natural caesarean: a woman-centred technique. BJOG. 2008;115: 1037-42.

21. Ministerio de Sanidad y Consumo. Encuesta Nacional de Salud 2006 [consultado el 12/09/ 2010]. Disponible en www.msps.es/estadEstudios/ estadisticas/encuestaNacional/encuesta2006.htm

22. Jovani Roda L, Gutiérrez Culsant P, Aguilar Martín C, Navarro Caballé T, Mayor Peguerotes I, Jornet Torrent $\mathrm{Y}$. Influencia del personal sanitario de asistencia primaria en la prevalencia de la lactancia materna. An Esp Pediatr. 2002;57:534-9.

23. Gibson MV, Díaz VA, Mainous AG, Geesey ME. Prevalence of breastfeeding and acculturation in Hispanics: results from NHANES 1999-2000 study. Birth. 2005;32:93-8.

24. Flacking $\mathrm{R}$, Nyqvist $\mathrm{KH}$, Ewald U. Effects of socioeconomic status on breastfeeding duration in mothers of preterm and term infants. Eur J Public Health. 2007; 17:579-84.

25. Olang B, Farivar K, Heidarzadeh A, Strandvik $B$, Yngve $A$. Breastfeeding in Iran: prevalence, duration and current. Recommendations. Int Breastfeed J. 2009;4:8.

26. Liu J, Rosenberg KD, Sandoval AP. Breastfeeding duration and perinatal cigarette smoking in a population-based cohort. Am J Public Health. 2006;96:309-14.

27. Weiser TM, Lin M, Garikapaty V, Feyerharm RW, Bensyl DM, Zhu BP. Association of maternal smoking status with breastfeeding practices: Missouri, 2005. Pediatrics. 2009;124:1603-10.

28. Hernández Aguilar MT, González Lombide $E$, Bustinduy Bascarán A, Arana Cañedo-Argüelles C, Martínez-Herrera Merino B, Blanco del Val A, y cols. Centros de Salud IHAN (Iniciativa de Humanización de la Atención al Nacimiento y la Lactancia). Una garantía de calidad. Rev Pediatr Aten Primaria. 2009;11:513-29. 